\title{
Application of modified homotopy perturbation method and amplitude frequency formulation to strongly nonlinear oscillators
}

\author{
Seyd Ghasem Enayati, M. Azimi, M. Jouya
}

Faculty of Mech, Babol University of Technology, Babol, Iran

Faculty of New Sciences and Technologies, University of Tehran, Tehran, Iran

Department of Mechnical Engineering, University of Siegen, Germany

Received: 12 January 2016, Accepted: 5 August 2016

Published online: 23 January 2017.

\begin{abstract}
In this paper, two powerful analytical methods known as modified homotopy perturbation method and Amplitude Frequency Formulation called respectively MHPM and AFF, are introduced to derive approximate solutions of a system of ordinary differential equations appear in mechanical applications. These methods convert a difficult problem into a simple one, which can be easily handled. The obtained solutions are compared with numerical fourth order runge-kutta method to show the applicability and accuracy of both MHPM and AFF in solving this sample problem. The results attained in this paper confirm the idea that MHPM and AFF are powerful mathematical tools and they can be applied to linear and nonlinear problems.
\end{abstract}

Keywords: Nonlinear oscillation, modified homotopy perturbation method, amplitude frequency formulation, analytical solution.

\section{Introduction}

Most of natural events and phenomena such as oscillation take place nonlinearly. Except for a few number of some nonlinear equations with solutions which are easy to find, solving these nonlinear problems can make researchers encounter difficulties in finding the exact analytical solution, thus it may guide authors to use various approximate analytical methods, such as Parameter expansion Method [1-3], Variational Iteration Method [4-11], Homotopy Perturbation Method [12-18], Amplitude Frequency Formulation [19-22], the Max-Min Approach [23-25], Modifed Homotopy Perturbation Method [26-29], energy Balance Method [30], Adomian Decomposition Method [31, 32], Differential Transformation Method [33, 34] and Amplitude Frequency Formulation [35].

In this paper, MHPM and MMA are used to solve three kinds of oscillators in the form,

$$
u^{\prime \prime}+f(u(t))=0
$$

where $\mathrm{u}$ and $\mathrm{t}$ respect to the generalized dimensionless displacement and time variable.

Modified homotopy perturbation method (MHPM) and Amplitude Frequency Formulation (AFF) suggested by J. H. He are striking methods to solve nonlinear oscillatory equations. The results obtained by these methods are valid for not only weakly nonlinear equations, but also strong ones. 


\section{Solution procedures}

\subsection{Basic idea of modified homotopy perturbation method}

To suggest the basic ideas of this method, the following equation is considered

$$
\ddot{u}+1 \cdot u=u-\Psi(\ddot{u}, \dot{u}, u, t)
$$

So, the following homotopy can be yield.

$$
\ddot{u}+1 \cdot u=p[u-\Psi(\ddot{u}, \dot{u}, u, t)], p \in[0,1] .
$$

Due to the alteration of homotopy parameter $\mathrm{p}$ from zero to the unity, when $p=0$, Eq. (3) turns into the linearalized equation $\ddot{u}_{0}+\omega^{2} u_{0}=0$ and when it's one, equation will be the original one.

The solution $u$ and 1 as coefficient of $u$ can be expanded as follows.

$$
\begin{aligned}
& u=\sum_{i=1}^{n} p^{i} \cdot u_{i} \\
& 1=\omega^{2}-\sum_{i=1}^{n} p^{i} \cdot \alpha_{i} .
\end{aligned}
$$

Replacing Eq. (4) and Eq. (5) into Eq. (3), and equating the terms with the identical powers of p, yields.

$$
\begin{aligned}
& p^{0}: \ddot{u}_{0}+\omega^{2} u_{0}=0 \\
& p^{1}: \ddot{u}_{1}+\omega^{2} u_{1}-N\left(u, \dot{u}_{0}, \ddot{u}_{0}, t\right)=0 .
\end{aligned}
$$

By inserting the answer of Eq. (6) $u_{0}=A \cos (\omega t)$ into Eq. (7), the following equation can be obtained.

$$
\ddot{u}_{1}+\omega^{2} u_{1}-\rho\left(A \cos (\omega t),-A \omega \sin (\omega t),-A \omega^{2} \cos (\omega t), t\right)=0 \text {. }
$$

Using Fourier series expansion, the secular term can be achieved.

$$
\begin{aligned}
& \rho(\omega t)=\sum_{n=0}^{\infty} b_{2 n+1} \cos [(2 n+1) \omega t] \approx b_{1} \cos (\omega t) \\
& b_{1}=\frac{4}{\pi} \int_{0}^{\frac{\pi}{2}} \rho(\omega t) d(\omega t) .
\end{aligned}
$$

In order to avoid the secular term the following equation should be considered.

$$
b_{1}=0
$$

Setting $\mathrm{p}=1$ in equation gives.

$$
1=\omega^{2}-\alpha_{1}
$$

So frequency $\omega$ can be yield. 


\subsection{Basic idea of Frequency Formulation}

For a generalized nonlinear oscillator in Eq. (1) two trial functions are considered as follows.

$$
\begin{aligned}
& u_{1}=A \cos (t) \\
& u_{2}=A \cos (\omega t) .
\end{aligned}
$$

Substituting Eq. (13) and Eq. (14) into Eq. (1) yields Residuals $R_{1}(t)$ and $R_{2}\left(t_{2}\right)$ where $t_{2}=\omega t$. Here weighted residuals can be introduced in the following form.

$$
\begin{aligned}
& \tilde{R}_{1}(t)=\frac{4}{T_{1}} \int_{0}^{\frac{T_{1}}{4}} R_{1}(t) \cos (t) d t, T_{1}=\frac{2 \pi}{\omega_{1}} \\
& \tilde{R}_{2}\left(t_{2}\right)=\frac{4}{T_{2}} \int_{0}^{\frac{T_{2}}{4}} R_{2}\left(t_{2}\right) \cos (\omega t) d t_{2}, T_{2}=\frac{2 \pi}{\omega_{2}} .
\end{aligned}
$$

According to He's frequency formulation the amplitude frequency formulation can be assumed.

$$
\omega^{2}=\frac{\omega_{1}^{2} \tilde{R}_{2}\left(t_{2}\right)-\omega_{2}^{2} \tilde{R}_{1}(t)}{\tilde{R}_{2}\left(t_{2}\right)-\tilde{R}_{1}(t)}
$$

where $\omega_{1}=1$ and $\omega_{2}=\omega$. Substituting Eq. (15) and Eq. (16) into Eq. (17), $\omega_{A F F}$ can be obtained.

\section{Applications of solution procedures}

\section{1 example}

In the first example physical model of nonlinear equation in the following figure is considered.

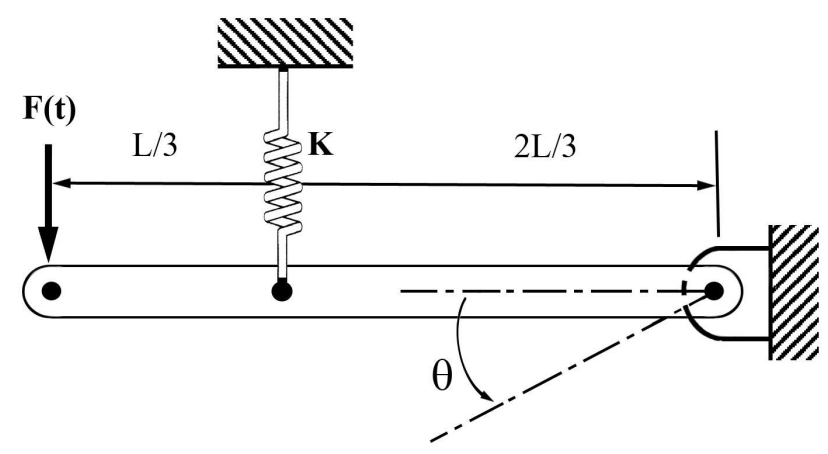

The equation of motion is written in the following form.

$$
\left(\frac{1}{3} m l^{2}\right) \ddot{\theta}+\frac{4}{9} k l^{2} \sin (\theta) \cos (\theta)=F(t) l \cos (\theta), \theta(0)=0, \dot{\theta}(0)=0
$$




\subsubsection{Applying MHPM to Example 1}

By choosing $\sin (\theta)=\theta-\frac{\theta^{3}}{3 !}+\frac{\theta^{5}}{5 !}$ and $\cos (\theta)=1-\frac{\theta^{2}}{2 !}+\frac{\theta^{4}}{4 !}$ Eq. (18) can be rewritten as follows.

$$
\ddot{\theta}+1 \theta=\frac{-4 k\left(\theta-\frac{2 \theta^{3}}{3}+\frac{2}{15} \theta^{5}\right)}{3 m}+\frac{3 F_{0} \sin \left(w_{0} t\right)\left(1-\frac{\theta^{2}}{2}+\frac{\theta^{4}}{24}\right)}{m l}+\theta .
$$

Using the homotopy parameter $p$ in Eq. (3), Following homotopy can be established as follows.

$$
\ddot{\theta}+1 \theta=p\left[\frac{-4 k\left(\theta-\frac{2 \theta^{3}}{3}+\frac{2}{15} \theta^{5}\right)}{3 m}+\frac{3 F_{0} \sin \left(w_{0} t\right)\left(1-\frac{\theta^{2}}{2}+\frac{\theta^{4}}{24}\right)}{m l}+\theta\right] .
$$

Replacing Eq. (4) and Eq. (5) into Eq. (20) and expanding that, first two linear equations can be written as follows.

$$
\begin{aligned}
& p^{0}: \ddot{\theta}_{0}+\omega^{2} \cdot \theta_{0}=0 \\
& p^{1}: \ddot{\theta}_{1}+\omega^{2} \theta_{1}=\alpha_{1} \theta_{0}-\frac{4 k\left(\theta_{0}-\frac{2}{3} \theta_{0}^{3}+\frac{2}{15} \theta_{0}^{5}\right)}{3 m}+\frac{3 F_{0} \sin \left(w_{0} t\right)\left(1-\frac{\theta_{0}^{2}}{2}+\frac{\theta_{0}{ }^{4}}{24}\right)}{m l}+\theta_{0} .
\end{aligned}
$$

Here $\theta_{0}=A \cos (\varpi t)$ can be acquire by solving Eq. (21) Substituting $\theta_{0}$ into Eq. (22) yields:

$$
\ddot{\theta}_{1}+\omega^{2} \theta_{1}=\rho(\omega t)
$$

where:

$$
\begin{aligned}
\rho(\omega t) & =\gamma_{1} A \cos \omega t+\rho(\omega t) \\
& =\gamma_{1} A \cos (\omega t)-\frac{4 k\left(A \cos (\omega t)-\frac{2}{3} A^{3} \cos (\omega t)^{3}+\frac{2}{15} A^{5} \cos (\omega t)^{5}\right)}{3 m} \\
& +\frac{3 F_{0} \sin \left(w_{0} t\right)\left(1-\frac{A^{2} \cos (\omega t)^{2}}{2}+\frac{A^{4} \cos (\omega t)^{4}}{24}\right)}{m l}+A \cos (\omega t) .
\end{aligned}
$$

Utilizing the following Fourier expansion series yields.

$$
\begin{gathered}
\rho(\omega t)=\sum_{n=0}^{\infty} \delta_{2 n+1} \cos [(2 n+1) \omega t]=\delta_{1} \cos (\omega t)+\delta_{3} \cos (3 \omega t)+\ldots \\
\delta_{1} \cong\left(\frac{4}{\pi} \int_{0}^{\frac{\pi}{2}} \rho(\phi) \cos (\phi) d \phi\right) \cos (\omega t) \\
=\frac{1}{45 \pi m l}\left(12 F_{0} A^{4} \sin \left(w_{0} t\right)-60 k A l \pi+30 k A^{3} l \pi-5 k A^{5} l \pi-180 F_{0} A^{2} \sin \left(w_{0} t\right)+540 F_{0} \sin \left(w_{0} t\right)\right)
\end{gathered}
$$

where,

$$
\begin{aligned}
\ddot{\theta}_{1}+\omega^{2} \theta_{1} & =\frac{1}{45 \pi m l}\left(\begin{array}{l}
12 F_{0} A^{4} \sin \left(w_{0} t\right)-60 k A l \pi+30 k A^{3} l \pi \\
-5 k A^{5} l \pi-180 F_{0} A^{2} \sin \left(w_{0} t\right)+540 F_{0} \sin \left(w_{0} t\right)
\end{array}\right) A \cos (\omega t) \\
& +\sum_{n=1}^{\infty} \delta_{2 n+1} \cos [(2 n+1) \omega t]
\end{aligned}
$$


In order to avoid secular term the following equation can be set.

$$
\delta_{1}=0
$$

Substituting $p=1$ into Eq. (5) gives.

$$
1=\gamma_{1}+\omega^{2}
$$

So the first approximation to the angular frequency is.

$$
\omega_{M H P M}=\frac{1}{15} \sqrt{5\left(\begin{array}{l}
\left(\frac{180 A-12 A^{3}}{m \pi l}\right) F_{0} \sin \left(w_{0} t\right) \\
+\frac{k}{m}\left(60-30 A^{2}+5 A^{3}\right)-\frac{540 F_{0} \sin \left(w_{0} t\right)}{A m \pi l}
\end{array}\right)} .
$$

3.1.2 Amplitude Frequency Formulation

In this section AFF is applied to solve Eq. (18). Here Eq. (18) can be written in the following form.

$$
\ddot{\theta}+a \theta+b \theta^{2}+c \theta^{3}+d \theta^{4}+\mathrm{e} \theta^{5}+f=0,
$$

where,

$$
\begin{aligned}
& a=\frac{4 k}{3 m} \quad b=\frac{3 F_{0} \sin \left(w_{0} t\right)}{2 m l} \\
& c=\frac{-8 k}{9 m} \quad d=\frac{3 F_{0} \sin \left(w_{0} t\right)}{24 m l} \\
& e=\frac{8 k}{45 m} \quad f=\frac{3 F_{0} \sin \left(w_{0} t\right)}{m l} .
\end{aligned}
$$

Replacing Eq. (13) and Eq. (14) as two trial functions into Eq. (18), gives the following Residuals.

$$
\begin{aligned}
& R_{1}(t)=-A \cos (t)+a A \cos (t)+b A^{2} \cos (t)^{2}+c A^{3} \cos (t)^{3}+d A^{4} \cos (t)^{4}+\mathrm{e} A^{5} \cos (t)^{5}+f \\
& R_{2}\left(t_{2}\right)=-A \cos (\omega t) \omega^{2}+a A \cos (\omega t)+b A^{2} \cos (\omega t)^{2}+c A^{3} \cos (\omega t)^{3}+d A^{4} \cos (\omega t)^{4}+\mathrm{e} A^{5} \cos (\omega t)^{5}
\end{aligned}
$$

Equating $\omega_{1}=1, \omega_{2}=\omega$, weighted residuals can be written in the following form.

$$
\begin{aligned}
\tilde{R}_{1}(t) & =\frac{2}{\pi} \int_{0}^{\frac{\pi}{2}}\left(\begin{array}{l}
-A \cos (t)+a A \cos (t)+b A^{2} \cos (t)^{2} \\
+c A^{3} \cos (t)^{3}+d A^{4} \cos (t)^{4}+\mathrm{eA}^{5} \cos (t)^{5}+f
\end{array}\right) \cos (t) d t \\
& =\frac{2\left(\frac{5}{32} \mathrm{e} \pi A^{5}-\frac{A \pi}{4}+\frac{8 d A^{4}}{15}+\frac{2 b A^{2}}{3}+\frac{3 c \pi A^{3}}{16}+\frac{a \pi A}{4}+f\right)}{\pi}
\end{aligned}
$$




\subsubsection{Amplitude frequency formulation}

Inserting Eq. (13) and Eq. (14) into Eq. (38), gives the following residuals.

$$
\begin{aligned}
\tilde{R}_{2}\left(t_{2}\right) & =\frac{2}{\pi} \int_{0}^{\frac{\pi}{2}}\left(\begin{array}{l}
-A \cos (\varphi) \omega^{2}+a A \cos (\varphi)+b A^{2} \cos (\varphi)^{2} \\
+c A^{3} \cos (\varphi)^{3}+d A^{4} \cos (\varphi)^{4}+\mathrm{e} A^{5} \cos (\varphi)^{5}
\end{array}\right) \cos (\varphi) d \varphi \\
& =\frac{2\left(\frac{5}{32} \mathrm{e} \pi A^{5}-\frac{A \omega^{2} \pi}{4}+\frac{8 d A^{4}}{15}+\frac{2 b A^{2}}{3}+\frac{3 c \pi A^{3}}{16}+\frac{a \pi A}{4}+f\right)}{\pi}
\end{aligned}
$$

Substituting Eq. (35) and Eq. (36) into Eq. (17) yields Amplitude-frequency equation.

$$
\omega_{A F F}=\frac{1}{60} \sqrt{30\left(120 a+75 \mathrm{e} A^{4}+\frac{256 d A^{3}+320 b A}{\pi}+\frac{480 f}{A \pi}+90 c A^{2}\right)} .
$$

Considering the following value of parameters, the comparison between numerical solution and analytical methods are illustrated in Fig.1 and Fig.2.

$$
k=1000 \frac{N}{m^{2}}, m=10 \mathrm{~kg}, l=1 m, F_{0}=1, w_{0}=1 .
$$

As shown below, amplitude frequency formulation and Modified homotopy perturbation method have a high validity in comparison with runge-kutta method.

$$
R_{1}(t)=-A \cos (t)+\frac{k_{1} A \cos (t)-F_{0} \sin \left(w_{0} t\right)}{m}+\frac{k_{2} A^{3} \cos (t)^{3}}{2 m h^{2}}
$$

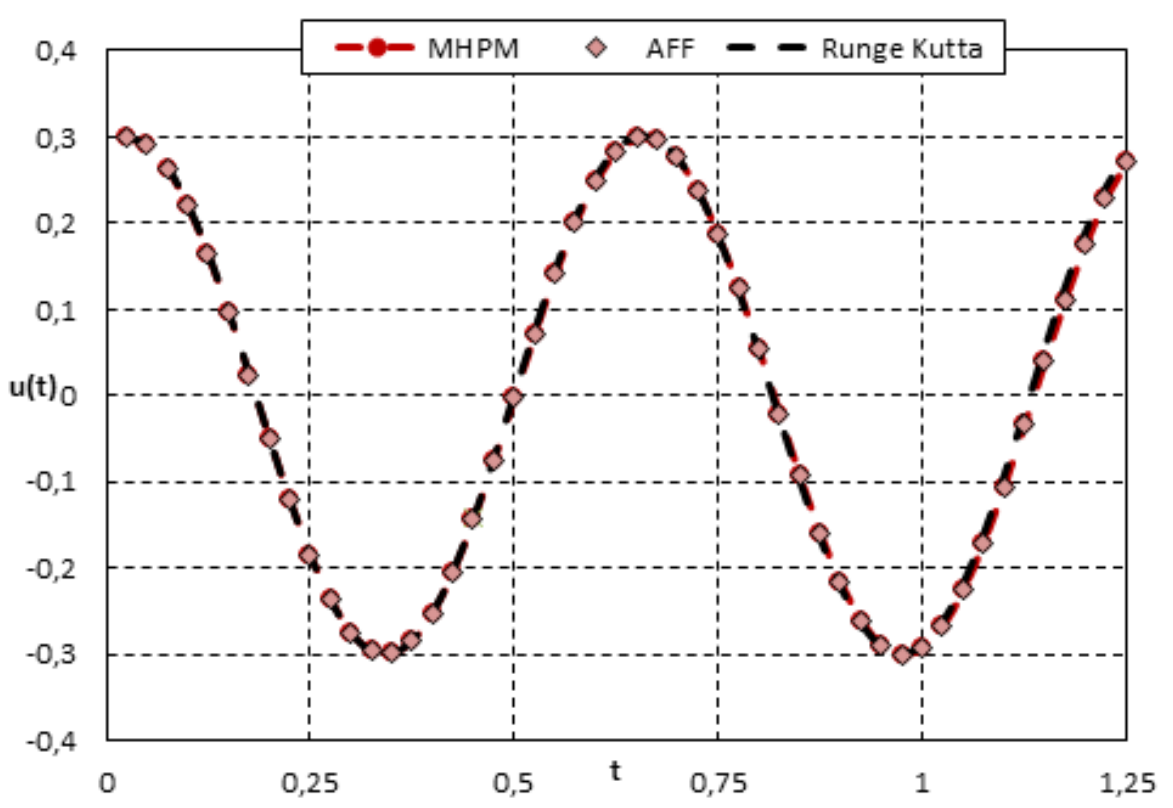

Fig. 1: Comparison of time history response between AFF \& MHPM \& forth order Runge-Kutta where $A=0.3$. 


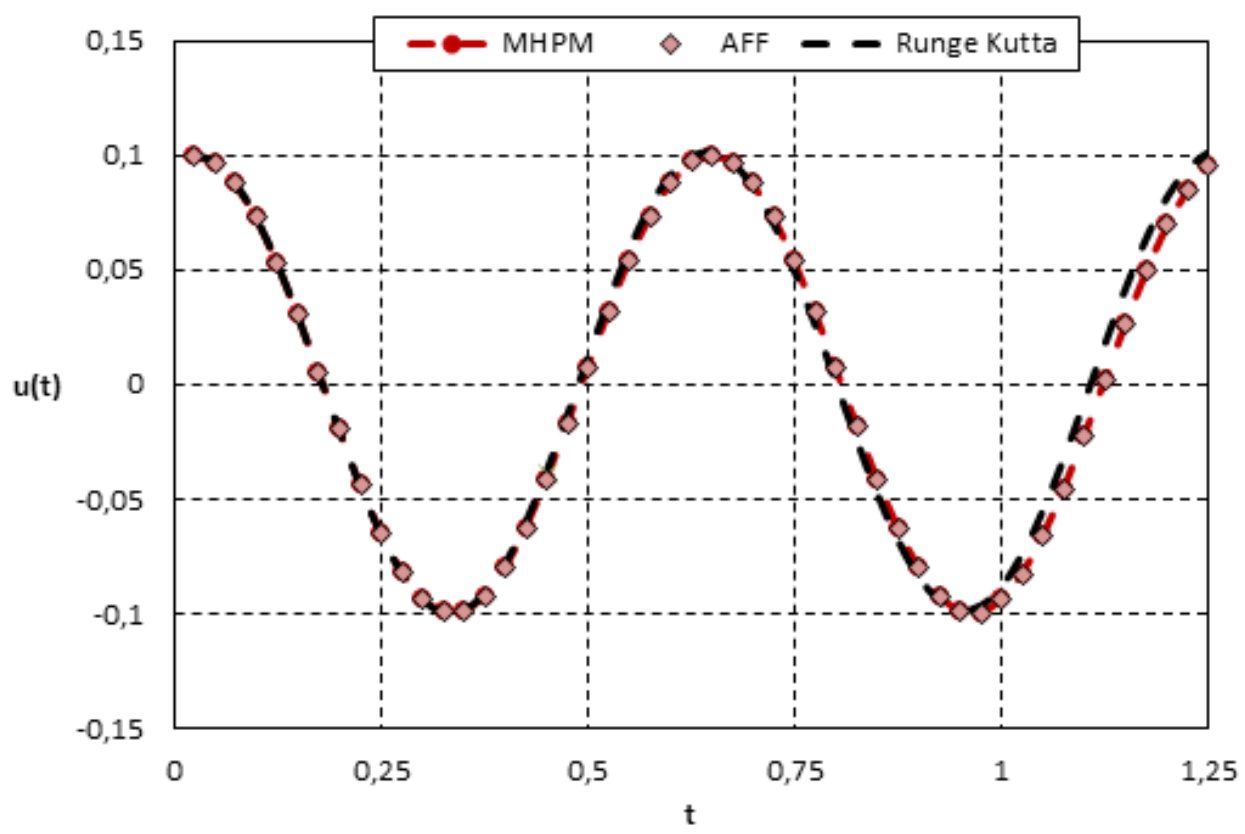

Fig. 2: Comparison of time history response between AFF \& MHPM \& forth order Runge-Kutta where $A=0.1$

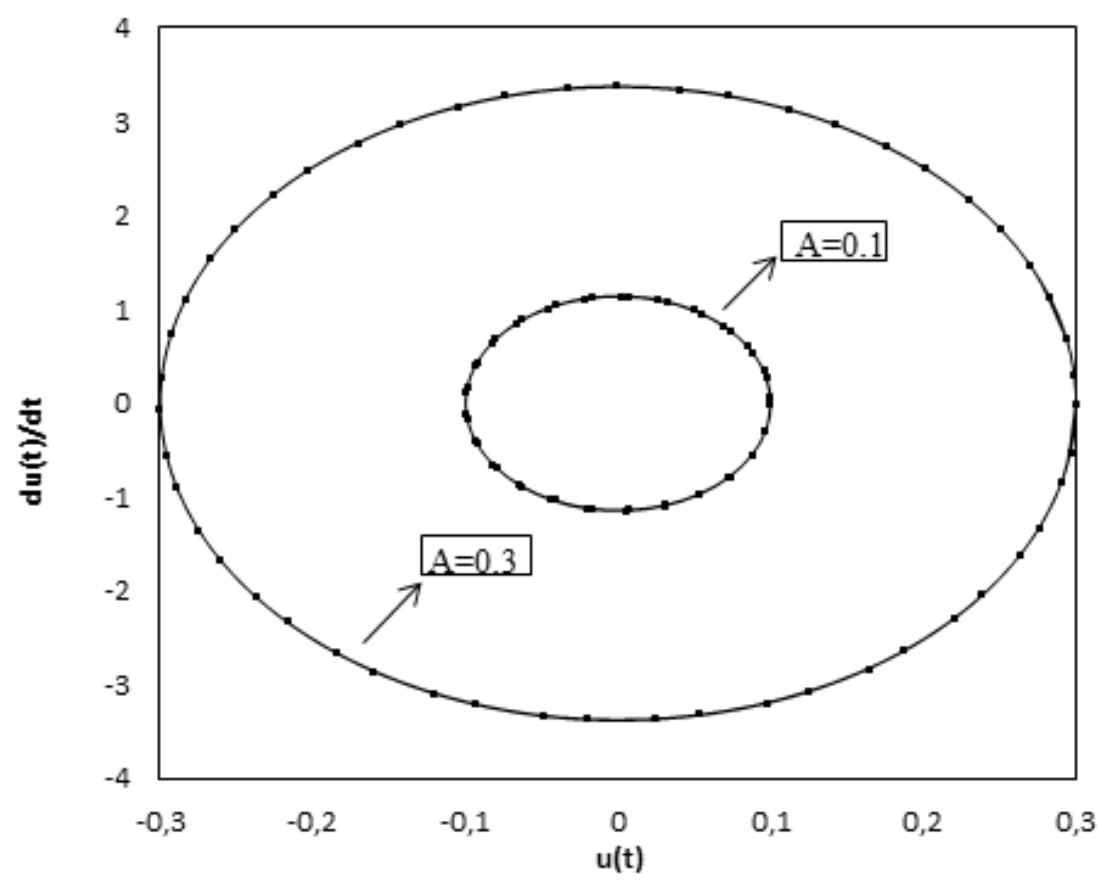

Fig. 3: Comparison of phase curve between two different initial conditions.

Example 1. In this example Duffing equation with constant coefficient is considered. 


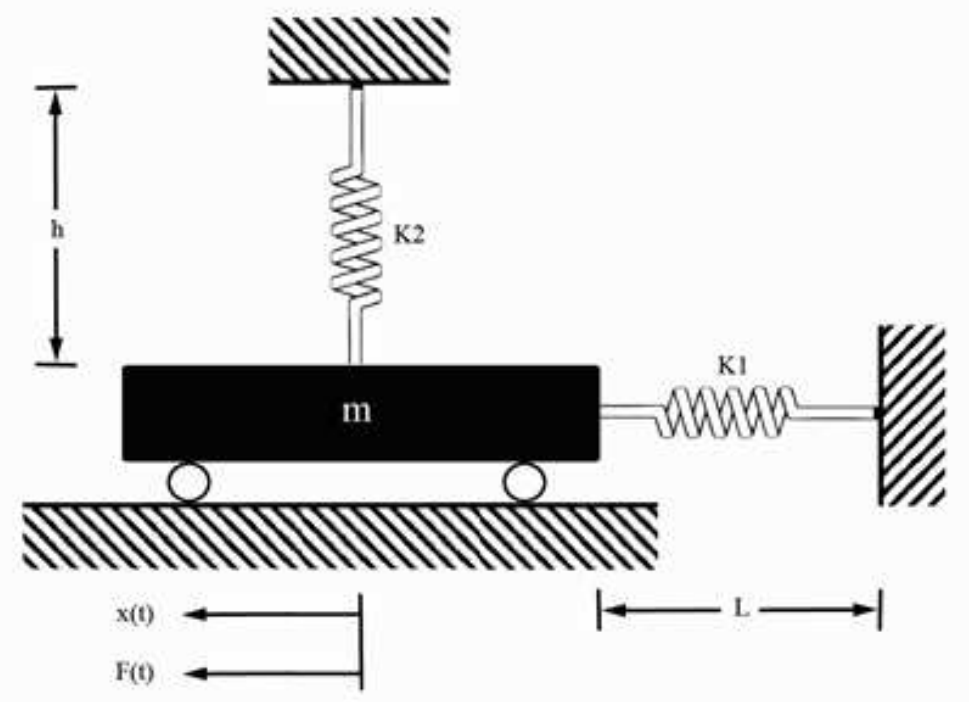

Fig. 4: Comparison of phase curve between two different initial conditions.

Equation of motion of the physical model of nonlinear equation in the following figure is written as follows.

$$
\ddot{x}+\frac{k_{1}}{m} x+\frac{k_{2}}{2 m h^{2}} x^{3}-\frac{F_{0} \sin \left(w_{0} t\right)}{m}=0, x(0)=A, \dot{x}(0)=0 .
$$

\subsection{Applying MHPM to example 2}

Eq. (38) can be rewritten in the following form.

$$
\ddot{x}+1 x=\left(1-\frac{k_{1}}{m}\right) x-\frac{k_{2}}{2 m h^{2}} x^{3}+\frac{F_{0} \sin w_{0} t}{m} .
$$

Utilizing the homotopy parameter $p$ in Eq.(3), Following homotopy can be identified as follows.

$$
\ddot{x}+1 x=p\left[\left(1-\frac{k_{1}}{m}\right) x-\frac{k_{2}}{2 m h^{2}} x^{3}+\frac{F_{0} \sin \left(w_{0} t\right)}{m}\right] .
$$

By substituting Eq. (4) and Eq. (5) into Eq. (40) and extending it, first two linear equations can be rewritten in the following form.

$$
\begin{gathered}
p^{0}: \ddot{x}_{0}+\omega^{2} \cdot x_{0}=0 \\
p^{1}: \ddot{x}_{1}+\omega^{2} x_{1}=\alpha_{1} x_{0}+\left(1-\frac{k_{1}}{m}\right) x_{0}-\frac{k_{2}}{2 m h^{2}} x_{0}^{3}+\frac{F_{0} \sin \left(w_{0} t\right)}{m} .
\end{gathered}
$$

Here $x_{0}=A \cos (\varpi t)$ can be obtained by solving Eq. (41). Substituting $x_{0}$ into Eq. (42) yields,

$$
\ddot{x}_{1}+\omega^{2} x_{1}=\rho(\omega t)
$$


where,

$$
\rho(\omega t)=\gamma_{1} A \cos (\omega t)+\left(1-\frac{k_{1}}{m}\right) A \cos (\omega t)-\frac{k_{2}}{2 m h^{2}} A^{3} \cos (\omega t)^{3}+\frac{F_{0} \sin \left(w_{0} t\right)}{m}
$$

By using the following Fourier expansion series.

$$
\begin{gathered}
\rho(\omega t)=\sum_{n=0}^{\infty} \delta_{2 n+1} \cos [(2 n+1) \omega t]=\delta_{1} \cos (\omega t)+\delta_{3} \cos (3 \omega t)+\ldots \\
\delta_{1} \operatorname{cong}\left(\frac{4}{\pi} \int_{0}^{\frac{\pi}{2}} \rho(\phi) \cos (\phi) d \phi\right) \cos (\omega t)=\frac{1}{8}\left(\frac{32 h^{2} F_{0} \sin w_{0} t-8 k_{1} A h^{2} \pi-3 k_{2} A^{3} \pi}{\pi m h^{2}}\right)+A \omega^{2}
\end{gathered}
$$

Now,

$$
\ddot{x}_{1}+\omega^{2} x_{1}=\left(\frac{1}{8}\left(\frac{32 h^{2} F_{0} \sin \left(w_{0} t\right)-8 k_{1} A h^{2} \pi-3 k_{2} A^{3} \pi}{\pi m h^{2}}\right)+A \omega^{2}\right) A \cos (\omega t)+\sum_{n=1}^{\infty} \delta_{2 n+1} \cos [(2 n+1) \omega t]
$$

For avoiding secular term the following equation should be determined.

$$
\delta_{1}=0
$$

Substituting $p=1$ into Eq. (5) yields.

$$
1=\gamma_{1}+\omega^{2} .
$$

So the first approximation to the angular frequency is.

$$
\begin{gathered}
\omega_{M H P M}=\sqrt{\frac{k_{1}}{m}+\frac{3 k_{2} A^{2}}{8 m h^{2}}-\frac{4 F_{0} \sin \left(w_{0} t\right)}{A m \pi}}, \\
R_{2}\left(t_{2}\right)=-A \cos (\omega t) \omega^{2}+\frac{k_{1} A \cos (\omega t)-F_{0} \sin \left(w_{0} t\right)}{m}+\frac{k_{2} A^{3} \cos (\omega t)^{3}}{2 m h^{2}} .
\end{gathered}
$$

As presented in previous example, following residuals can be obtained as follows,

$$
\begin{aligned}
\tilde{R}_{1}(t) & =\frac{2}{\pi} \int_{0}^{\frac{\pi}{2}}\left(-A \cos (t)+\frac{k_{1} A \cos (t)-F_{0} \sin \left(w_{0} t\right)}{m}+\frac{k_{2} A^{3} \cos (t)^{3}}{2 m h^{2}}\right) \cos (t) d t \\
& =\frac{8 k_{1} A \pi h^{2}-32 h^{2} F_{0} \sin \left(w_{0} t\right)-8 A \pi m h^{2}+3 k_{2} A^{3} \pi}{16 \pi m h^{2}}
\end{aligned}
$$

$$
\begin{aligned}
\tilde{R}_{2}\left(t_{2}\right) & =\frac{2}{\pi} \int_{0}^{\frac{\pi}{2}}\left(-A \cos (\varphi) \omega^{2}+\frac{k_{1} A \cos (\varphi)-F_{0} \sin \left(w_{0} t\right)}{m}+\frac{k_{2} A^{3} \cos (\varphi)^{3}}{2 m h^{2}}\right) \cos (\varphi) d \varphi \\
& =\frac{8 k_{1} A \pi h^{2}-32 h^{2} F_{0} \sin \left(w_{0} t\right)-8 \omega^{2} A \pi m h^{2}+3 k_{2} A^{3} \pi}{16 \pi m h^{2}}
\end{aligned}
$$


Replacing Eq. (63) and Eq. (64) and solving that, Amplitude-frequency relationship can be obtained in the following form:

$$
\omega_{A F F}=\sqrt{\frac{k_{1}}{m}+\frac{3 k_{2} A^{2}}{8 m h^{2}}-\frac{4 F_{0} \sin \left(w_{0} t\right)}{A m \pi}}
$$

Considering the following value of parameters, comparison between numerical solution and analytical methods are illustrated in Fig.3 and Fig.4.

$$
h=0.5, m=10(K g), k_{1}=1000, k_{2}=1100, F_{0}=1, w_{0}=1, l=1(m) .
$$

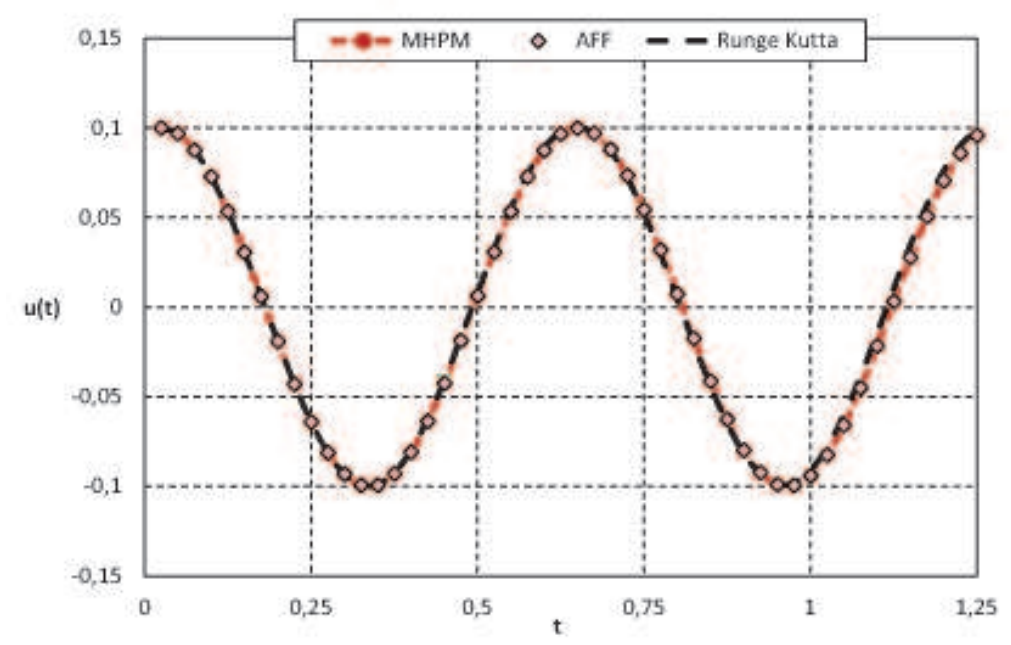

Fig. 5: comparison of time history response between AFF \& MHPM \& forth order runge-kutta, where $A=0.1$.

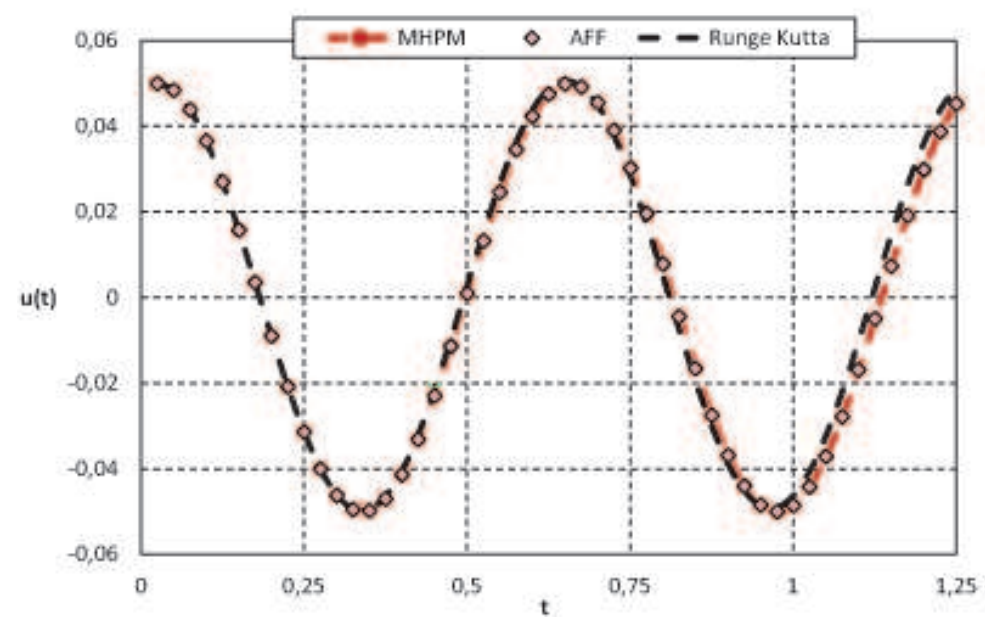

Fig. 6: comparison of time history response between AFF \& MHPM \& forth order runge-kutta, where $A=0.05$. 


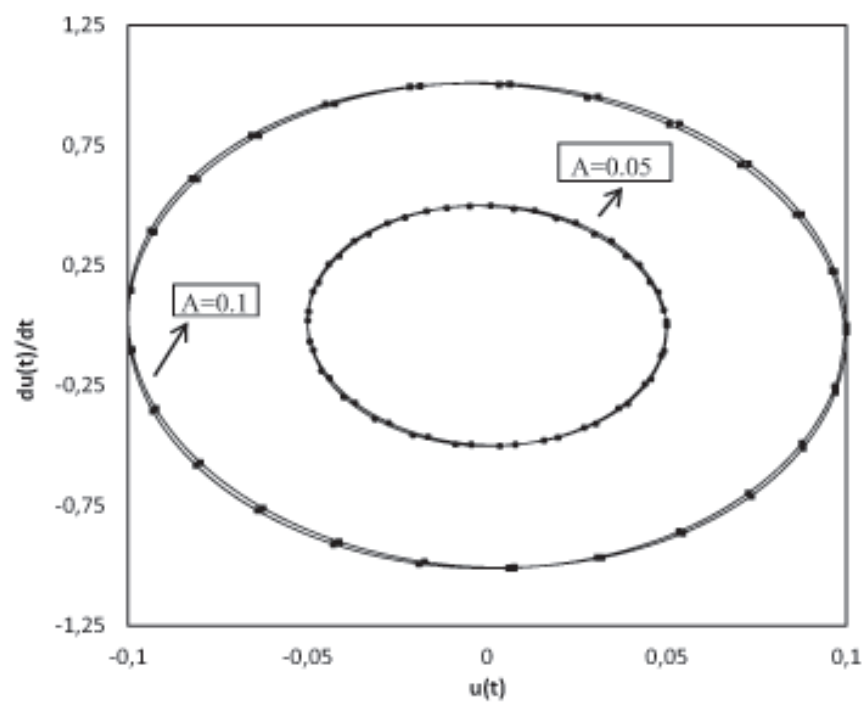

Fig. 7: Comparison of phase curve between two different initial conditions.

\subsection{Example 3}

The physical model of nonlinear oscillation is clarified in the following figure,

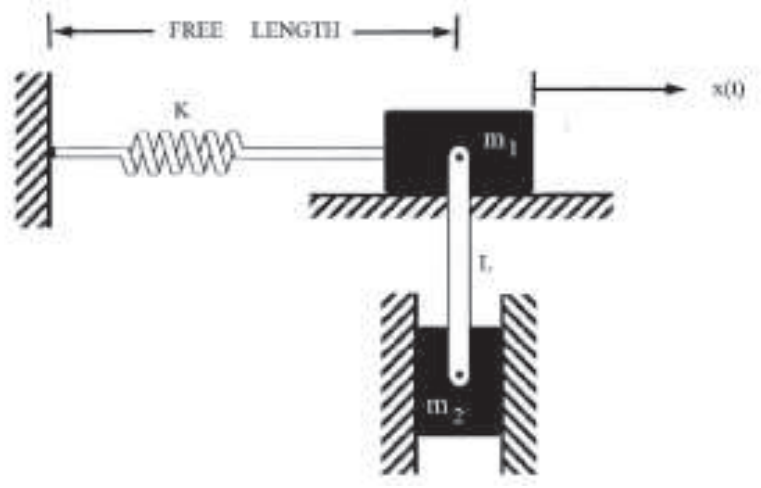

The equation of motion can be yield as,

$$
\left(m_{1}+\frac{m_{2} x^{2}}{l^{2}-x^{2}}\right) \ddot{x}+\frac{m l x \dot{x}^{2}}{\left(l^{2}-x^{2}\right)}+k x+m_{2} g \frac{x}{\sqrt{\left(l^{2}-x^{2}\right)}}=0
$$

where $g$ is the Gravitational acceleration, Let $u=\frac{x}{l}$ then expanding for $|u|<<1$, gives,

$$
\left(1+\frac{m_{2}}{m_{1}} u^{2}\right) \ddot{u}+\left(\frac{m_{2}}{m_{1}}\right) u \dot{u}^{2}+\omega_{0}^{2} u+\frac{m_{2} g}{2 l m_{1}} u^{3}+\cdots=0
$$

where

$$
\omega_{0}^{2}=\frac{k}{m_{1}}+\frac{m_{2} g}{l m_{1}}
$$


Equation (2) can be rewritten in the following form,

$$
\ddot{u}+1 \cdot u=a u^{2} \ddot{u}+a u \dot{u}^{2}+(1-b) u+c u^{3} .
$$

where $a=\frac{m_{2}}{m_{1}}, \quad b=\omega_{0}^{2}, c=\frac{m_{2} g}{2 l m_{1}}$.

\subsubsection{Applying MHPM to example 3}

Using the homotopy parameter $\mathrm{p}$ in above equation, the following homotopy can be established,

$$
\ddot{u}+1 \cdot u=p\left[a u^{2} \ddot{u}+a u \dot{u}^{2}+(1-b) u+c u^{3}\right] .
$$

Inserting Eq. (4) and Eq. (5) into Eq. (71), the linear equations can be written as follows,

$$
\begin{aligned}
& p^{0}: \ddot{u}_{0}+\omega^{2} u_{0}=0 \\
& p^{1}: \ddot{u}_{1}+\omega^{2} u_{1}-\alpha_{1} u_{0}+a u_{0}^{2} \ddot{u}_{0}+a u_{0} \dot{u}_{0}^{2}+c u_{0}^{3}+(1-b) u_{0}=0 .
\end{aligned}
$$

Solving Eq. (73) gives,

$$
u_{0}=A \cos (\omega t)
$$

Substituting Eq. (74) into Eq. (73), obtains,

$$
\begin{aligned}
\ddot{u}_{1}+\omega^{2} u_{1}= & \alpha_{1} A \cos (\omega t)+a A^{3} \cos (\omega t)^{3} \omega^{2}-a A^{3} \cos (\omega t) \sin (\omega t)^{2} \omega^{2} \\
& -c A^{3} \cos (\omega t)^{3}-(1-b) A \cos (\omega t) .
\end{aligned}
$$

Fourier expansion series expansion, gives,

$$
\begin{gathered}
\alpha_{1} A \cos (\omega t)+a A^{3} \cos (\omega t)^{3} \omega^{2}-a A^{3} \cos (\omega t) \sin (\omega t)^{2} \omega^{2} \\
-c A^{3} \cos (\omega t)^{3}-(1-b) A \cos (\omega t)=\sum_{n=0}^{\infty} \delta_{2 n+1} \cos [(2 n+1) \omega t] \approx \delta_{1} \cos (\omega t) . \\
\delta_{1}=\frac{4}{\pi} \int_{0}^{\frac{\pi}{2}}\left(\begin{array}{l}
\alpha_{1} A \cos (\varphi)+a A^{3} \cos (\varphi)^{3} \omega^{2}-a A^{3} \cos (\varphi) \sin (\varphi)^{2} \omega^{2} \\
-c A^{3} \cos (\varphi)^{3}-(1-b) A \cos (\varphi)
\end{array}\right) d \varphi \\
=\frac{A}{4}\left(2 a A^{2} \omega^{2}+4 \alpha_{1}+4-3 c A^{2} \cdot-4 b\right)
\end{gathered}
$$

Using Eq. (77) and avoiding secular term $\delta_{1}=0$,the first approximation of frequency can be obtained as follows:

$$
\omega_{M H P M}=\frac{1}{2} \frac{\sqrt{\left(2+a A^{2}\right)\left(8 \omega_{0}^{2}+3 c A^{2}\right)}}{2+a A^{2}}
$$

Substituting Eq.(70) into Eq.(79) yields,

$$
\omega_{M H P M}=\frac{1}{2} \sqrt{\frac{\left(8 \omega_{0}^{2} l m_{1}+3 m_{2} g A^{2}\right)}{l\left(m_{2} A^{2}+2 m_{1}\right)}}
$$




\subsubsection{Amplitude frequency formulation}

Similarly using Eq. (13) and Eq. (14) for Eq. (66), yields the following Residuals.

$$
\begin{aligned}
R_{1}(t)= & -A \cos (t)-A^{3}\left(\frac{m_{2}}{m_{1}}\right) \cos (t)^{3}+A^{3}\left(\frac{m_{2}}{m_{1}}\right) \cos (t) \sin (t)^{2} \\
& +A \omega_{0}^{2} \cos (t)+A^{3}\left(\frac{m_{2} g}{2 l m_{1}}\right) \cos (t)^{3} \\
R_{2}\left(t_{2}\right)= & -A \cos (\omega t) \omega^{2}-A^{3}\left(\frac{m_{2}}{m_{1}}\right) \cos (\omega t)^{3} \omega^{2}+A \omega_{0}^{2} \cos (\omega t) \\
& +A^{3}\left(\frac{m_{2}}{m_{1}}\right) \cos (\omega t) \sin (\omega t)^{2} \omega^{2}+A^{3}\left(\frac{m_{2} g}{2 l m_{1}}\right) \cos (\omega t)^{3} .
\end{aligned}
$$

Locating at $\omega_{1}=1, \omega_{2}=\omega$ in Eq. weighted residuals can be obtained as follows,

$$
\begin{aligned}
& \tilde{R}_{1}(t)=\frac{2}{\pi} \int_{0}^{\frac{\pi}{2}}\left(-A \cos (t)-A^{3}\left(\frac{m_{2}}{m_{1}}\right) \cos (t)^{3}+A^{3}\left(\frac{m_{2}}{m_{1}}\right) \cos (t) \sin (t)^{2}\right. \\
& \left.+A \omega_{0}^{2} \cos (t)+A^{3}\left(\frac{m_{2} g}{2 l m_{1}}\right) \cos (t)^{3}\right) \cos (t) d t \\
& =\frac{1}{2} A \omega_{0}^{2}+\frac{3}{8} A^{3}\left(\frac{m_{2} g}{2 l m_{1}}\right)-\frac{1}{4}\left(\frac{m_{2}}{m_{1}}\right) A^{3}-\frac{1}{2} A . \\
& \tilde{R}_{2}\left(t_{2}\right)=\frac{2}{\pi} \int_{0}^{\frac{\pi}{2}}\left(\begin{array}{l}
-A \cos (\varphi) \omega^{2}-A^{3}\left(\frac{m_{2}}{m_{1}}\right) \cos (\varphi)^{3} \omega^{2}+A \omega_{0}^{2} \cos (\varphi) \\
+A^{3}\left(\frac{m_{2}}{m_{1}}\right) \cos (\varphi) \sin (\varphi)^{2} \omega^{2}+A^{3}\left(\frac{m_{2} g}{2 l m_{1}}\right) \cos (\varphi)^{3}
\end{array}\right) \cos (\varphi) d \varphi \\
& =-\frac{1}{2} A \omega^{2}+\frac{3}{8}\left(\frac{m_{2} g}{2 l m_{1}}\right) A^{3}-\frac{1}{4} A^{3}\left(\frac{m_{2}}{m_{1}}\right) \omega^{2}+\frac{1}{2} \omega_{0}^{2} A .
\end{aligned}
$$

Substitution of Eq. (82) and Eq. (83) into Eq. (17) yields angular frequency as follows,

$$
\omega^{2}=\frac{\frac{1}{2} \omega_{0}^{2} A+\frac{3}{8}\left(\frac{m_{2} g}{2 l m_{1}}\right) A^{3}-\frac{1}{4} A^{3}\left(\frac{m_{2}}{m_{1}}\right) \omega^{2}-\frac{1}{2} A \omega^{2}-\omega^{2}\left(\frac{1}{2} \omega_{0}^{2} A+\frac{3}{8}\left(\frac{m_{2} g}{2 l m_{1}}\right) A^{3}-\frac{1}{4}\left(\frac{m_{2}}{m_{1}}\right) A^{3}-\frac{1}{2} A\right)}{-\frac{1}{4} A^{3}\left(\frac{m_{2}}{m_{1}}\right) \omega^{2}-\frac{1}{2} A \omega^{2}+\frac{1}{4}\left(\frac{m_{2}}{m_{1}}\right) A^{3}+\frac{1}{2} A}
$$

Solving Eq. (84), Amplitude-frequency relationship can be obtained,

$$
\omega_{A F F}=\frac{1}{2} \sqrt{\frac{8 \omega_{0}^{2} l m_{1}+3 m_{2} g A^{2}}{l\left(m_{2} A^{2}+2 m_{1}\right)}}
$$

Considering the following value of parameters, comparison between numerical solution and analytical methods are illustrated in Fig.5 and Fig.6.

$$
g=9.81 \mathrm{~m} / \mathrm{s}^{2}, k=100 \mathrm{~N} / \mathrm{m}^{2}, m_{1}=5 \mathrm{~kg}, m_{2}=1 \mathrm{~kg}, l=1 \mathrm{~m} .
$$

As shown below, amplitude frequency formulation and Modified homotopy perturbation method have a high validity in comparison with Runge-Kutta method. 


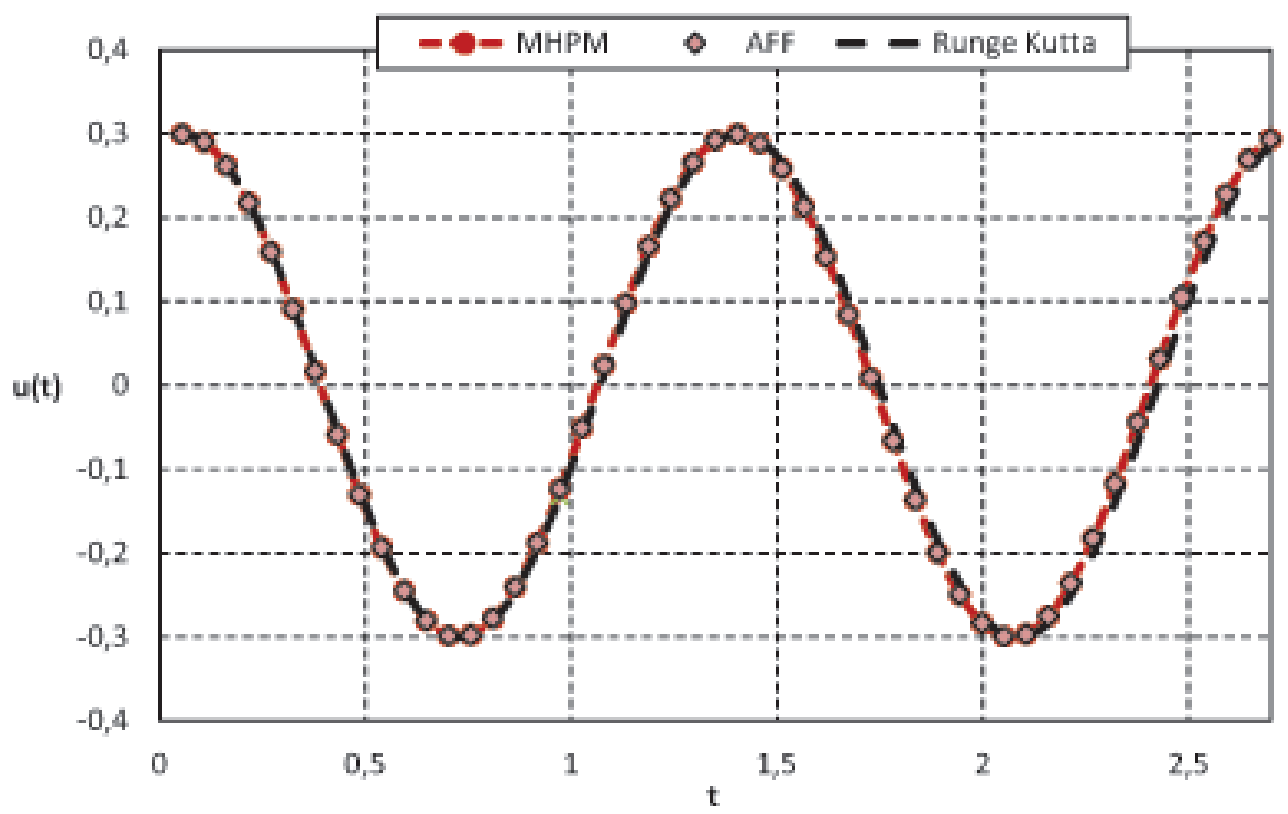

Fig. 8: Comparison of time history response between AFF \& MHPM \& forth order Runge-Kuttawhere, where $A=0.3$.

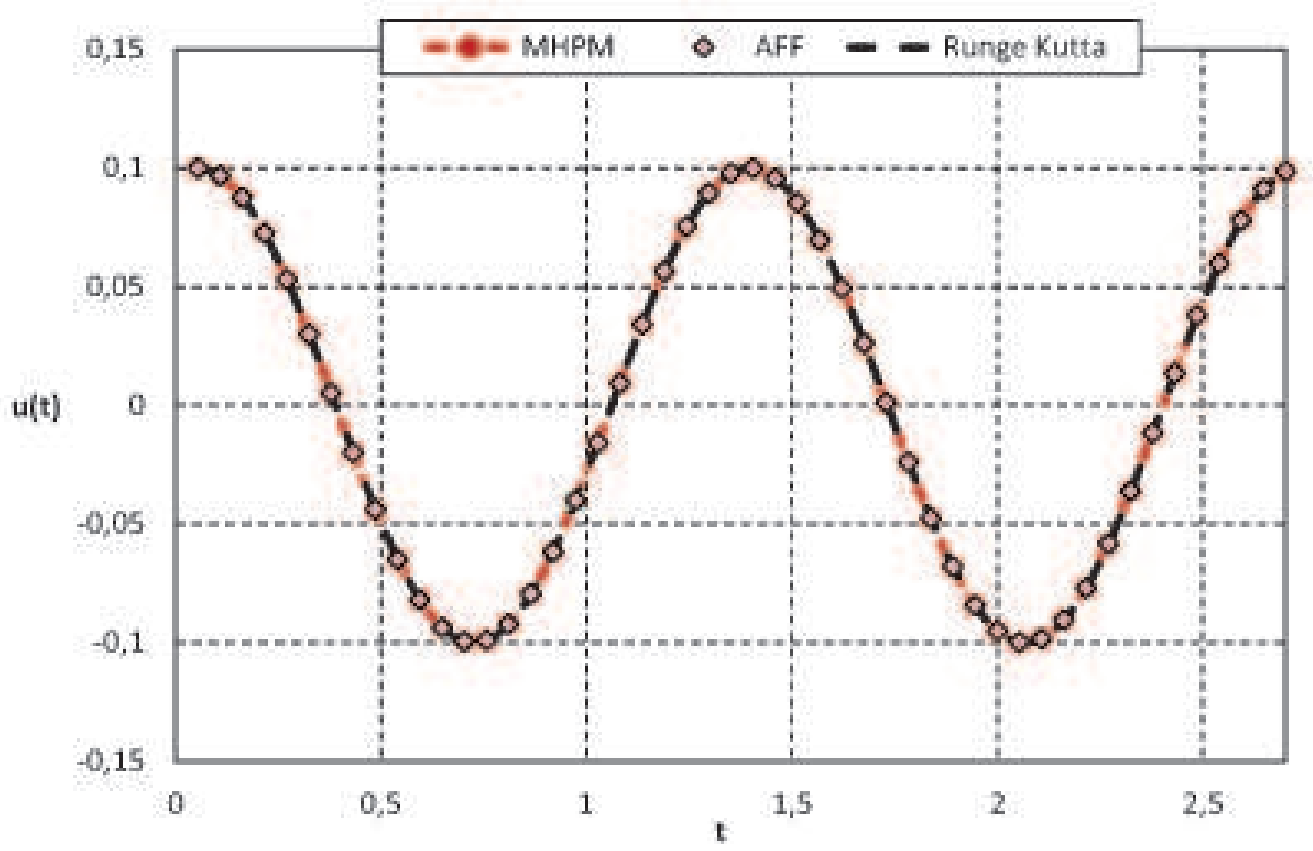

Fig. 9: Comparison of time history response between AFF \& MHPM \& forth order Runge-Kutta, where $A=0.1$. 


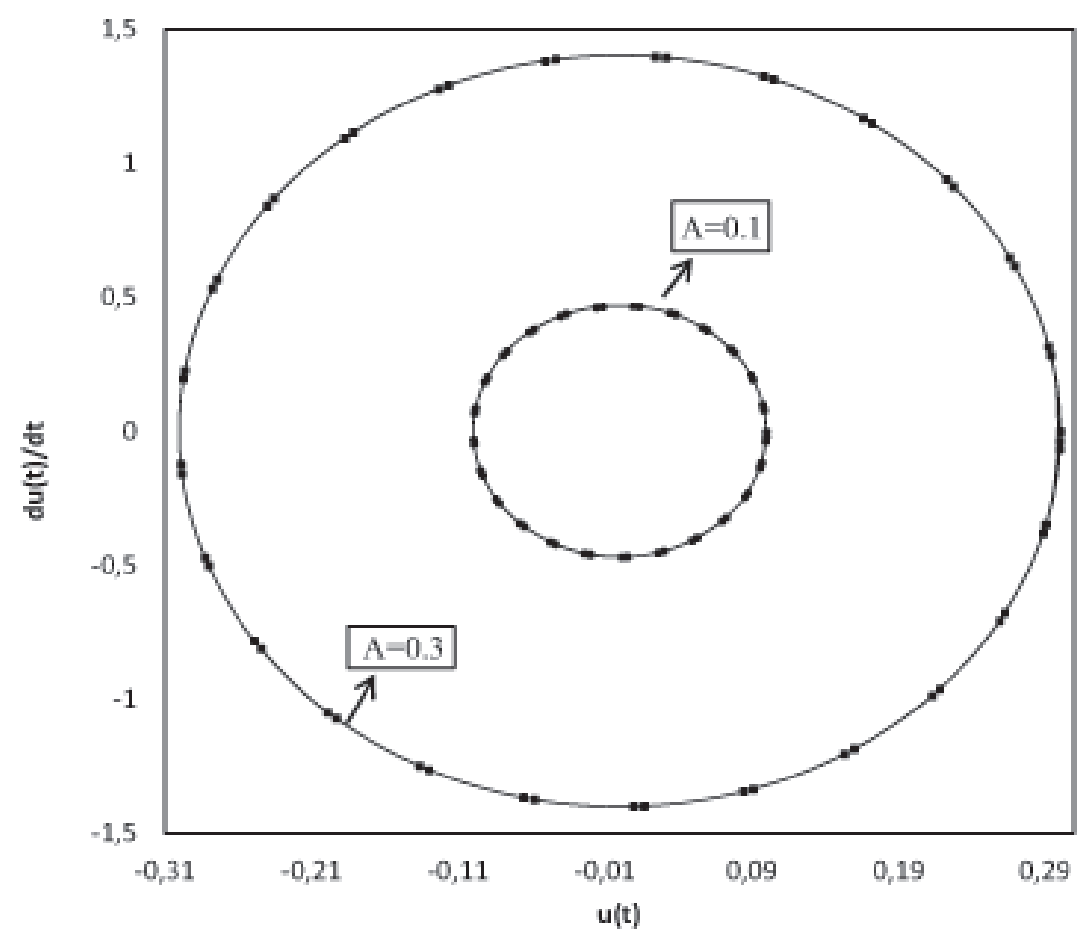

Fig. 10: Comparison of phase curve between two different initial conditions

\section{Conclusion}

In this paper, Modified Homotopy Perturbation Method (MHPM) and Amplitude Frequency Formulation (AFF) which are proved to be powerful mathematical tools to study nonlinear vibrating equations have been successfully developed and tested on three examples of nonlinear vibrating equations. The obtained results demonstrate that both methods are accurate, capable and convergent techniques and that they compare extremely well with numerical solution. According to Figures associated with above examples which are the comparison between analytical methods and numerical RungeKutta method of order 4, indicates that these methods provide highly precise answers for nonlinear equations. These examples illustrate the efficiency of the modified homotopy perturbation method and Amplitude Frequency Formulation and also it has been shown that these methods don't have any requirement for advanced calculus.

\section{Competing interests}

The authors declare that they have no competing interests.

\section{Authors' contributions}

All authors have contributed to all parts of the article. All authors read and approved the final manuscript.

\section{References}

[1] L.Xu, Determination of limit cycle by He's parameter-expanding method for strongly nonlinear oscillators, Journal of Sound and Vibration 302, 178_184 (2007). 
[2] L.Xu, Application of He's parameter-expansion method to an oscillation of a mass attached to a stretched elastic wire, Physics Letters A 368, 259-262 (2007).

[3] A. Kimiaeifar, A.R. Saidi, A.R. Sohouli, D.D. Ganji, Analysis of modified Van der Pol's oscillator using He's parameter-expanding methods, Current Applied Physics 10, 279_283 (2010).

[4] Ji-Huan He, Xu-Hong Wu, Variational iteration method, New development and applications, Computers and Mathematics with Applications 54, 881_894 (2007).

[5] D.D. Ganji, G.A. Afrouzi, R.A. Talarposhti, Application of variational iteration method and homotopy perturbation method for nonlinear heat diffusion and heat transfer equations, Physics Letters A 368, 450_457 (2007).

[6] L. Xu, Variational approach to solution of nonlinear dispersive $K(m, n)$ equation, Chaos, Solitons and Fractals 37 (1), $137 \_143$ (2008).

[7] L. Xu, Variational principles for coupled nonlinear Schrödinger equations, Physics Letters A 359, 627_629 (2006).

[8] D.D. Ganji, A. Sadighi, I. Khatami, Assessment of two analytical approaches in some nonlinear problems arising in engineering sciences, Physics Letters A 372, 4399_4406 (2008).

[9] Xumei Chen, Linjun Wang, The variational iteration method for solving a neutral functional-differential equation with proportional delays, Computers and Mathematics with applications 59, 2696_2702 (2010).

[10] Saeed Rastegar, Bahram Azizollah Ganji, Mojtaba Varedi , Mehran Erza, Application of He's variational iteration method to the estimation of diaphragm deflection in MEMS capacitive microphone, Measurement 44, 113_120 (2011).

[11] Ji-Huan He, An elementary introduction to the homotopy perturbation method, Computers and Mathematics with Applications 57, 410_412 (2009).

[12] A Belendez,T Belendez, A Marquez, C Neipp, Application of He's homotopy perturbation method to conservative truly nonlinear oscillators. Chaos, Solitons and Fractals 37, 770_80 (2008).

[13] A. Beléndez, A. Hernández, T.Beléndez, C. Neipp, A. Márquez, Higher accuracy analytical approximations to a nonlinear oscillator with discontinuity by He's homotopy perturbation method, Physics Letters A 372, 2010-2016 (2008).

[14] Augusto Beléndez, Homotopy perturbation method for a conservative $x^{1 / 3}$ force nonlinear oscillator, Computers \& Mathematics with Applications 58, 2267-2273 (2009).

[15] M. Gorji-Bandpy, M. Azimi, and M. Mostofi, 2011, Analytical Methods to a Generalized Duffing Oscillator, Australian Journal of Basic and Applied Science, Vol. 5, No. 11, pp. 788-796.

[16] S.M. Moghimi, D.D. Ganji, H. Bararnia, M. Hosseini, M. Jalaal, Homotopy perturbation method for nonlinear MHD JefferyHamel problem, Computers and Mathematics with Applications 61, 2213_2216 (2011).

[17] Songxin Liang, David J.Jeffrey, Comparison of homotopy analysis method and homotopy perturbation method through an evolution equation, Communications in Nonlinear Science and Numerical Simulation, 4057_4064 (2009).

[18] J.H.He, An improved amplitude frequency formulation for nonlinear oscillators, International Journal of Nonlinear Sciences and Numerical Simulation 9, 211_212 (2008).

[19] L. Geng and X. C. Cai, He's frequency formulation for nonlinear oscillators, European Journal of Physics 28, 923 _931 (2007).

[20] Ling Zhao, He's frequency_amplitude formulation for nonlinear oscillators with an irrational force, Computers and Mathematics with Applications 58, 2473_2476, (2009).

[21] S.S. Ganji, D.D.Ganji, M.G.Sfahani, S.Karimpour, Application of AFF and HPM to the systems of strongly nonlinear oscillation, Current Applied Physics 10, 1317-1325 (2010).

[22] J. H. He, Max-Min Approach to Nonlinear Oscillators, International Journal of Nonlinear Sciences and Numerical Simulation 9 , 207_210 (2008).

[23] De-Qiang Zeng, Nonlinear oscillator with discontinuity by the max_min approach, Chaos, Solitons and Fractals 42 (2009) 28852889.

[24] S.S. Ganji, A. Barari, D.D. Ganji, Approximate analysis of two-mass-spring systems and buckling of a column, Computers and Mathematics with Applications 61,1088_1095 (2011).

[25] Xinlong Feng, Yinnian He, Modified homotopy perturbation method for solving the Stokes equations, Computers and Mathematics with Applications 61, 2262_2266 (2011).

[26] A. Beléndez , C. Pascual, S. Gallego, M. Ortuño, C. Neipp, Application of a modified He's homotopy perturbation method to obtain higher-order approximations of an $x^{1 / 3}$ force nonlinear oscillator, Physics Letters A 371, 421-426 (2007).

[27] A. Beléndez, C. Pascual, T. Beléndez, A. Hernández, Solution for an anti-symmetric quadratic nonlinear oscillator by a modified He's homotopy perturbation method, Nonlinear Analysis: Real World Applications 10, 416-427 (2009). 
[28] Junfeng Lu, An analytical approach to the sine Gordon equation using the modified homotopy perturbation method, Computers and Mathematics with Applications 58, 2313_2319 (2009).

[29] Davood D Ganji, Mohammadreza Azimi, Mehdi Mostofi, Energy Balance Method and amplitude frequency formulation based of strongly nonlinear oscillator, indian journal of pure \& applied physics, Vol. 50, 2012, pp. 670-675.

[30] D. D. Ganji, M. Sheikholeslami, and H. R. Ashorynejad, Analytical Approximate Solution of Nonlinear Differential Equation Governing Jeffery-Hamel Flow with High Magnetic Field by Adomian Decomposition Method, ISRN Mathematical Analysis Volume 2011 (2011).

[31] A. Sadighi, D.D. Ganji, Analytic treatment of linear and nonlinear Schrödinger equations: A study with homotopy-perturbation and Adomian decomposition methods, Physics Letters A 372, 465_469 (2008).

[32] D.D.Ganji, M.Azimi, Application of DTM on MHD Jeffery Hamel Problem with Nanoparticle, 2013, The Scientific Bulletin Series D, vol. 75, pp.223-230.

[33] M. Azimi, D. D. Ganji, F. Abbassi, Study on MHD Viscous Flow over a Stretching Sheet Using DTM-Pade' Technique, Modern Mechanical Engineering, Vol. 2, No. 4, 2012, pp. 126-129.

[34] D.D.Ganji, M.Azimi, Application of Max min Approach and Amplitude Frequency Formulation to the nonlinear oscillation systems, 2012, The Scientific Bulletin Series A, Issue.3, pp.131-140. 